\title{
Utility-Based User Grouping and Bandwidth Allocation for Wireless Multicast Systems
}

\author{
Juan Liu*, Wei Chen ${ }^{\dagger}$, Member, IEEE, Zhigang Cao ${ }^{\dagger}$, Senior Member, IEEE, \\ Ying Jun (Angela) Zhang ${ }^{\ddagger}$, Member, IEEE, Soung Chang Liew ${ }^{\ddagger}$, Senior Member, IEEE \\ ${ }^{*}$ State Key Laboratory on Microwave and Digital Communications \\ Tsinghua National Laboratory for Information Science and Technology (TNList) \\ Department of Electronic Engineering, Tsinghua University, Beijing 100084, China \\ $\ddagger$ Department of Information Engineering, The Chinese University of Hong Kong \\ Email:*liujuan05@mails.thu.edu.cn ${ }^{\dagger}\{$ wchen, czg-dee $\} @$ thu.edu.cn ${ }^{\ddagger}\{$ yjzhang, soung $\} @$ ie.cuhk.edu.hk
}

\begin{abstract}
With the proliferation of wireless multimedia applications, multicast/broadcast has been recognized as an efficient technique to transmit a large volume of data to multiple mobile stations at the same time. In most multicast systems, the transmitter (e.g. base station) adapts its data rate to the furthest located users, so as to guarantee service quality to as many users as possible. Predictably, the more users in a multicast group, the lower data rate the base station can transmit. On the other hand, grouping more users together leads to a more efficient utilization of spectrum bandwidth, as these users are served simultaneously. This bring the interesting problem that presses for solution: how to group users in a cell into multicast groups and how to allocate a fixed amount of bandwidth resource to the groups, to achieve a good balance between throughput and fairness in multicast systems. In this paper, we formulate the united user grouping and bandwidth allocation strategy into a utility-based optimization problem. One method of signomial programming is used to solve the non-convex optimization problem. Numerical results will show that this suboptimal algorithm performs well even compared to the optimal one. Moreover, through theoretical analysis, we prove that the best user grouping and bandwidth allocation scheme of throughput maximization is to allocate the entire bandwidth to the unique group containing the users located within a ring-shaped region with an optimal outer radius $r^{*}$.
\end{abstract}

\section{INTRODUCTION}

The explosive growth of wireless broadband multimedia services has placed an unprecedented demand on the already scarce wireless spectrum bandwidth [1]. Hence, efficient utilization of the scarce spectrum resource has become a key challenge in the design of next-generation wireless systems. It is worth noting that many emerging applications such as mobile TV and group oriented mobile commerce aim to deliver the same large volume of data to multiple users in the network [2]. Such applications are particularly well suited for multicast transmission, which broadcasts the same content to a group of users at a single transmission. Conceivably, point-to-multipoint multicast transmission can greatly improve the spectrum efficiency compared to the point-to-point unicast transmission. As a matter of fact, multicast/broadcast features have been included in the recent release of IEEE 802.16e standard [3], which adopts Orthogonal Frequency Division

This work was supported by NSFC founded key project under Grant No. 60832008, and NSFC/RGC jointly funded project under Grant No. 60618001, and Chuanxin Funding.
Multiplexing (OFDM) as the air interface to combat multipath fading effect [4].

In wireless networks, multicast transmission to a group of users benefits from the broadcast nature of radio channels. The capacity region of broadcast channels was first derived in [5]. The authors of [6] [7] investigated the capacity region and the optimal power allocation scheme for fading broadcast channels. These literatures show that to maximize the users' aggregate throughput, each user of a Multicast Group (MG) should be allocated different data rate and transmission power according to their diverse channel conditions. In most multicast systems, a Base Station (BS) transmits to the users of a MG at a same data rate for simplicity. Recently, some algorithms for scheduling and resource allocation are presented to periodically adjust the data transmission rate to each MG. Sharif et al proposed three scheduling schemes with the rational rate constraint on MGs to maximize the system throughput [8]. Proportional fairness multicast scheduling algorithms were proposed by Won et al to select an appropriate multicast data rate and a group for transmission in each slot for cellular data networks [9]. Suh et al in [10] studied the subcarrier/bit allocation problem and proposed optimal/suboptimal algorithms for wireless multicast multicarrier systems. We have investigated the grouping scheme for the users requiring the same program to maximize the average throughput in the Rayleigh fading environment, when the transmission rate is adapted to the users with the worst channel condition in each MG [11].

In this paper, we consider multicast in access networks where a BS or access point (AP) broadcasts data to a group of users. To ensure the signal reception quality for as many users as possible, the data transmission rate is adapted to the furthest users in each MG because the user's reception SNR usually decreases with the distance apart from the BS. Predictably, the data transmission rate decreases with the radial location of users in a group. On the other hand, in a system with a fixed amount of bandwidth resource, including the radially wider distributed users into a MG leads to a larger group and thus a more efficient utilization of system bandwidth. With the two factors counteracting each other, it is important to understand the following question: how to form MGs and 


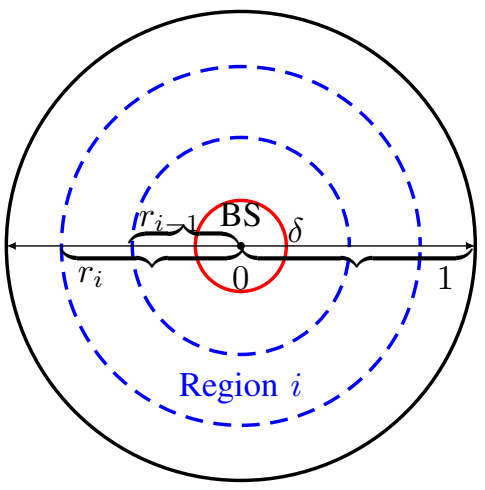

Fig. 1. In a unit-radius cell, all the target users are divided into $N$ MGs. The users of $\mathfrak{m}_{i}$ are located in Region $i$, a ring-shaped region with inner radius $r_{i-1}$ and outer radius $r_{i}$

how to allocate the fixed system bandwidth to the groups to balance excellently between throughput and fairness for the users receiving the same contents in a cell. The answer to this question would serve as a guideline to scheduling transmissions in wireless multicast systems. To the best of our knowledge, such problem has not be investigated before.

Assuming that a large number of users requiring the same program are uniformly distributed in the cell and divided into finite MGs, we know from our prior work [11] that the average throughput of each group approaches a constant asymptotically. We attempt to find the User Grouping and Bandwidth Allocation (UGBA) scheme that determines the user location and fixed bandwidth share of each MG. This UGBA problem will be formulated into a utility maximization problem. The utility functions play an important role in this paper as they usually do to model network protocols, applications etc. in [12], [13]. A method of Signomial Programming (SGP) is used to find a suboptimal solution of this non-convex problem. The numerical results show that the SGP algorithm performs well in finding a suitable UGBA scheme. From numerical simulations, we know that in the severer fading environments (larger cells etc.), the users should be divided into more MGs. We also prove that the best UGBA scheme to maximize the average throughput is to allocate the whole bandwidth to a unique user group, within a region with an optimal ring radius.

The rest of this paper is organized as follows. Section II introduces the system model. In Section III, the utilitybased UGBA problem is formulated and a SGP method is described to get the suboptimal solution. We introduce the best UGBA scheme of throughput maximization in Section IV. The simulation results and conclusions are presented respectively in Section V and Section VI.

\section{SySTEM MODEL}

\section{A. UGBA Model}

We consider multicast OFDM systems, where the users requiring the same program are distributed in a unit-radius circular cell. The user distribution is denoted by $f(r, \theta)$, where $r$ and $\theta$ denote the radial and angular coordinate respectively.
Assume that the smallest separation between the BS and the Mobile Station (MS) is denoted by a small constant $\delta$. The users are divided into $N$ MGs according to their radial coordinates. As shown in Fig. 1, each group $i$ includes the users with $r_{i-1} \leq r \leq r_{i}\left(r_{0}=\delta, r_{N}=1\right)$ that consist a user set denoted by $S_{i}$. The number of users in this group can be evaluated by $K_{i}=\int_{r_{i-1}}^{r_{i}} \int_{0}^{2 \pi} f(r, \theta) r d r d \theta$. They share the same bandwidth that is denoted by $\alpha_{i} B$, where $0 \leq \alpha_{i} \leq 1$ is the proportion of the bandwidth allocated to MG $i$ and $B$ is the entire system bandwidth for all groups.

In a cell, the channel (power) gain of user $m$ on a specific subcarrier is represented by $\zeta_{m}=l_{m} f_{m}$, where $l_{m}$ denotes the path loss and $f_{m}$ denotes the small-scale fading respectively. Typically, given the path loss exponent $\rho$, the path loss $l_{m}=d_{m}{ }^{-\rho}$ presents how the received signal strength decays with the distance $d_{m}$ apart from the BS. The user's average received power can be approximately computed by $\bar{P}_{m}=P_{0}\left(d_{m} / d_{0}\right)^{-\rho}$, where $P_{0}$ is the average received signal power at the reference point with the distance $d_{0}$. The user's small-scale fading $f_{m}$ is traditionally formulated as a random variable with the exponential distribution (Rayleigh fading). Without loss of generality, we assume $\mathbf{E}\left(f_{m}\right)=1$. Each user's channel gains on different sub-carriers are also independent.

As mentioned before, the transmission rate to each MG is adapted to the furthest user in this group. For each user $m \in S_{i}$ with $r_{i-1} \leq r \leq r_{i}$, it is reasonably assumed that the equivalent average received power is $\bar{P}_{i}=P_{0}\left(r_{i} / d_{0}\right)^{-\rho}$, and the equivalent instantaneous SNR is $\gamma_{m}=\frac{P_{0}}{N_{0} B_{c}}\left(r_{i} / d_{0}\right)^{-\rho} f_{m}$, where $N_{0}$ denotes the single-sided power spectral density of Additive White Gaussian Noise (AWGN). Based on Shannon's channel capacity, the transmission rate to $\mathrm{MG} i$ can be represented by $R_{i}=\min _{m \in S_{i}} B_{c} \log \left(1+\gamma_{m}\right)$, where $B_{c}$ is the bandwidth of each subcarrier. Under the assumption of the stationary and ergodic channels, the user's average throughput equals to $\bar{R}_{i}=\mathbf{E}\left(\alpha_{i} N_{s} R_{i}\right)$, where $N s=B / B_{c}$ is the number of the overall sub-carriers. From Theorem 2 of [11], the user's average throughput is given by

$$
\bar{R}_{i}=\mathbf{E}\left(\alpha_{i} N_{s} R_{i}\right)=\frac{\alpha_{i} \bar{P}_{i}}{N_{0}}=\alpha_{i} \frac{P_{0}}{N_{0}}\left(r_{i} / d_{0}\right)^{-\rho} \text { nats } / \mathrm{s},
$$

when $K_{i} \rightarrow \infty$ and $N s \rightarrow \infty$.

\section{B. Utility Maximization}

The UGBA problem is formulated into a utility maximization problem represented by

$$
\begin{aligned}
& \max _{r_{i}, \alpha_{i}} \frac{1}{K} \sum_{i=1}^{N} \sum_{m \in S_{i}} U\left(\bar{R}_{m}\right)=\frac{1}{K} \sum_{i=1}^{N} K_{i} U\left(\bar{R}_{i}\right) \\
& \text { subject to }\left\{\begin{array}{l}
\sum_{i=1}^{N} \alpha_{i}=1, \alpha_{i} \geq 0 \\
\delta=r_{0} \leq r_{1} \leq \cdots \leq r_{N}=1
\end{array}\right.
\end{aligned}
$$

where $U(\cdot)$ denotes the user's utility. Our objective is to maximize the user's average utility by optimizing $\vec{\alpha}=$ $\left(\alpha_{1}, \cdots, \alpha_{N}\right)$ and $\vec{r}=\left(r_{0}, r_{1}, \cdots, r_{N}\right)$. The first constraint 
means the entire system bandwidth should be allocated. The second constraint indicates the radial range of each MG.

To simplify the optimization problem further, two assumptions are introduced: a) a very large number of users are uniformly distributed in the cell so that $f(r, \theta)=\varphi$ is a sufficiently large constant for any $r$ and $\theta$; b) the system bandwidth $B$ is extraordinarily large. The group size of MG $i$ $K_{i}=\int_{r_{i-1}}^{r_{i}} \int_{0}^{2 \pi} f(r, \theta) r d r d \theta=\pi \varphi\left(r_{i}^{2}-r_{i-1}^{2}\right)$ also becomes sufficiently large. By the same token, the number of overall users is $K=\pi \varphi\left(1-\delta^{2}\right)$. In the following, the average data rate in (1) is substituted based on the above two assumptions. Clearly, it facilitates our further theoretical discussion. To be noted, it also has practical values, in that simulation results in [11] have shown that the average throughput approaches its asymptotic value when hundreds of users are included in a group and allocated hundreds of sub-carriers.

Substituting $\bar{R}_{i}$ and $K_{i}$ into the original problem (2) yields

$$
\begin{aligned}
& \max _{r_{i}, \alpha_{i}} \frac{1}{1-\delta^{2}} \sum_{i=1}^{N}\left(r_{i}^{2}-r_{i-1}^{2}\right) U\left(\alpha_{i} \frac{P_{0}}{N_{0}}\left(r_{i} / d_{0}\right)^{-\rho}\right) \\
& \text { subject to }\left\{\begin{array}{l}
\sum_{i=1}^{N} \alpha_{i}=1, \alpha_{i} \geq 0 \\
\delta=r_{0} \leq r_{1} \leq \cdots \leq r_{N}=1
\end{array}\right.
\end{aligned}
$$

Here, we consider the following utility function that is often applied in the literature

$$
U(x)=\left\{\begin{array}{ll}
x^{1-\kappa} /(1-\kappa) & \kappa \geq 0, \kappa \neq 1 \\
\log (x) & \kappa=1
\end{array},\right.
$$

where $x$ denotes a user's data rate. The parameter $\kappa$ reflects the tradeoff between the fairness among users and the utilization of resources. In this case of $\kappa=0$, the problem of utility maximization corresponds to that of throughput maximization. As $\kappa$ becomes large, the users' rate vector $\vec{r}$ satisfying utility maximization converges to that of max-min fairness. In the next sections, we will solve this optimization problem for different $\kappa$ to achieve the corresponding UGBA policies.

\section{UTILITY MAXIMIZATION}

In this section, the UGBA scheme to maximize the average utility is analyzed. First, we try to find the solution of (3) with $\kappa>1$ is described in detail. Here, from (4), the average utility function of (3) is $\overline{\mathcal{U}}=c_{1} \sum_{i=1}^{N}\left(r_{i}^{2}-r_{i-1}^{2}\right)\left(\alpha_{i}^{-1} r_{i}^{\rho}\right)^{\kappa-1}$, where $c_{1}=\frac{d_{0}^{\rho(1-\kappa)}}{\left(1-\delta^{2}\right)(1-\kappa)}\left(P_{0} / N_{0}\right)^{1-\kappa}$.

Theorem 1: At the optimal solution $\left(\vec{\alpha}^{*}, \vec{r}^{*}\right)$ to (3), we have $\alpha_{i}^{*}=\frac{f_{i}^{* b}}{\sum_{i=1}^{N} f_{i}^{* b}}$, where $b=\frac{1}{2(\kappa-1)}, f_{i}(\vec{r})=\left(r_{i}^{2}-\right.$ $\left.r_{i-1}^{2}\right) r_{i}^{\rho(\kappa-1)}$ and $f_{i}^{*}=f_{i}\left(\vec{r}^{*}\right)$.

Proof: By applying Hölder's inequality, for any $\vec{\alpha}$ and $\vec{r}$ we have $\overline{\mathcal{U}}_{1}=\sum_{i=1}^{N} \frac{f_{i}(\vec{r})}{\alpha_{i}^{\kappa-1}} \geq\left(\sum_{i=1}^{N} f_{i}(\vec{r})^{1 / \kappa}\right)^{\kappa}=\overline{\mathcal{U}}_{1}^{\prime}$, where $\sum_{i=1}^{N} \alpha_{i}=1$ is used. The inequality becomes an equality if and only if $\frac{f_{1}(\vec{r})^{1 / \kappa}}{\alpha_{1}^{2(\kappa-1) / \kappa}}=\cdots=\frac{f_{N}(\vec{r})^{1 / \kappa}}{\alpha_{N}^{2(\kappa-1) / \kappa}}$. We get
$\overline{\mathcal{U}}_{1}=\overline{\mathcal{U}}_{1}^{\prime}$ as $\alpha_{i}=\frac{f_{i}(\vec{r})^{b}}{\sum_{i=1}^{N} f_{i}(\vec{r})^{b}}$. In this case, the problem of maximizing $\overline{\mathcal{U}}=c_{1} \overline{\mathcal{U}}_{1}$ is equivalent to that of minimizing $\overline{\mathcal{U}}_{1}^{\prime}$ for the negative $c_{1}$. Then, it is also equivalent to the following minimization problem for $\kappa>1$

$$
\begin{aligned}
& \min _{r_{i}} \overline{\mathcal{U}}_{2}=\sum_{i=1}^{N}\left(r_{i}^{2}-r_{i-1}^{2}\right)^{\frac{1}{\kappa}} r_{i}^{\frac{\rho(\kappa-1)}{\kappa}} . \\
& \text { subject to } \delta=r_{0} \leq r_{1} \leq \cdots \leq r_{N}=1
\end{aligned}
$$

Let $\vec{r}^{*}$ and $\overline{\mathcal{U}}_{2}^{*}$ be the optimal solution and the optimal value of (5). For any $\vec{\alpha}$ and $\vec{r}$, we have $\overline{\mathcal{U}}_{1} \geq \overline{\mathcal{U}}_{1}^{\prime} \geq$ $\overline{\mathcal{U}}_{2}^{* \kappa}$ and thus $\overline{\mathcal{U}}=c_{1} \overline{\mathcal{U}}_{1} \leq c_{1} \overline{\mathcal{U}}_{2}^{* \kappa}$. When $\alpha_{i}^{*}=\frac{f_{i}^{* b}}{\sum_{i=1}^{N} f_{i}^{* b}}$ with $f_{i}^{*}=f_{i}\left(\vec{r}^{*}\right),\left(\vec{\alpha}^{*}, \vec{r}^{*}\right)$ is an optimal solution to the original utility maximization problem.

Now, we only need to find the solution of (5) so as to find the optimal solution $\left(\vec{\alpha}^{*}, \vec{r}^{*}\right)$ that we are pursuing. Since the minimization problem (5) is not convex, it's very difficult to obtain its optimal solution. Fortunately, with $\beta_{i}=\left(r_{i-1} / r_{i}\right)^{2}$, the problem (5) changes into the following optimization problem in the form of a SGP

$$
\begin{aligned}
& \min _{\beta_{i}} \overline{\mathcal{U}}_{2}=c_{3} \sum_{i=1}^{N}\left(1-\beta_{i}\right)^{\frac{1}{\kappa}} \prod_{j=1}^{i} \beta_{j}^{-\frac{\rho(\kappa-1)+2}{2 \kappa}}, \\
& \text { subject to } \prod_{i=1}^{N} \beta_{i}=\delta^{2}, \beta_{i} \leq 1
\end{aligned}
$$

where $c_{3}=\delta^{\frac{\rho(\kappa-1)+2}{\kappa}}$ is a constant. Here, a method described in [14] is applied. This method works well in practice with a good starting guess $\vec{\beta}^{(0)}$ since the problem is similar to a GP except the negative coefficient in the terms $1-\beta_{i}(\forall i)$. At each step, we replace the original objective and constraint functions with their local monomial approximations near the current guess $\vec{\beta}^{(k)}$. The SGP of each step thus becomes a GP that can be solved efficiently due to its convexity after the logarithmic transformation.

Next, an interesting property about the optimal solution of the problem (6) is presented in Theorem 2.

Theorem 2: For any $N_{1}<N_{2}$, the inequality $\overline{\mathcal{U}}_{2}^{*(1)} \geq$ $\overline{\mathcal{U}}_{2}^{*(2)}$ holds, where $\overline{\mathcal{U}}_{2}^{*(i)}$ is the optimal value of (6) with $N=N_{i}(i=1,2)$.

Proof: Suppose $\vec{\beta}^{*(1)}$ is an optimal solution of (6) with $N=N_{1}$, and correspondingly $\overline{\mathcal{U}}_{2}^{*(1)}$ is the optimal value. With $N_{1}<N_{2}$, we can construct a vector $\vec{\beta}^{(2)}=\left(\vec{\beta}^{*(1)}, \vec{\nu}\right)$ where $\vec{\nu}$ is a vector of all $N_{2}-N_{1}$ ones. Clearly, it is a solution of (6) with $N=N_{2}$. Accordingly, the objective function satisfies $\overline{\mathcal{U}}_{x}=\overline{\mathcal{U}}_{2}^{*(1)} \geq \overline{\mathcal{U}}_{2}^{*(2)}$. Thus we have $\overline{\mathcal{U}}_{2}^{*(1)} \geq \overline{\mathcal{U}}_{2}^{*(2)}$ for any $N_{1}<N_{2}$.

From [14], there is an implicit constraint that the variables are positive, i.e., $\beta_{i}>0$. It also means that each $\beta_{i}$ in (6) are not allowed to equal to 1 . Here, $\overline{\mathcal{U}}_{2}^{*(i)}$ and $\overline{\mathcal{U}}_{2}^{S(i)}$ respectively denote the optimal value and the suboptimal value obtained by the method of SGP with $N=i$ in (6). If $\overline{\mathcal{U}}_{2}^{*(i)}=\overline{\mathcal{U}}_{2}^{*(j)}$ exists for $i<j$, we get $\overline{\mathcal{U}}_{2}^{*(i)}<\overline{\mathcal{U}}_{2}^{S(j)}$. In this case, it's necessary 
to compare the suboptimal values of (6) with $N=1, \ldots N_{x}$ so as to get an almost best UGBA scheme for $N=N_{x}$.

In the same way, the problem (3) with $0<\kappa<1$ becomes the following maximization problem

$$
\begin{aligned}
& \max _{\chi_{i}, \beta_{i}} \overline{\mathcal{U}}_{3}=c_{3} \sum_{i=1}^{N} \chi_{i}^{\frac{1}{\kappa}} \prod_{j=1}^{i} \beta_{j}^{-\frac{\rho(\kappa-1)+2}{2 \kappa}} \\
& \text { subject to }\left\{\begin{array}{l}
\beta_{i}+\chi_{i} \leq 1, \forall i \\
\prod_{i=1}^{N} \beta_{i}=\delta^{2}, \beta_{i} \leq 1
\end{array}\right.
\end{aligned}
$$

The suboptimal solution of (7) can similarly be obtained through the SGP algorithm as stated above. Moreover, from the definition of proportional fair in [13], it is reasonable to approximate a UGBA scheme for $\kappa=1$ by searching the solution of (6) or (7) for $\kappa \rightarrow 1$.

\section{Throughrut MAXimization}

As mentioned in Section II, when $\kappa=0$, the utility maximization problem turns out to be a throughput maximization problem. The average throughput of (3) can be represented by $\overline{\mathcal{C}}=\frac{1}{1-\delta^{2}} \frac{P_{0}}{N_{0} d_{0}^{-\rho}} \sum_{i=1}^{N}\left(r_{i}^{2}-r_{i-1}^{2}\right) \alpha_{i} r_{i}^{-\rho}$. With variable substitutions $\beta_{i}=\left(r_{i-1} / r_{i}\right)^{2}$, this optimization problem becomes

$$
\begin{aligned}
& \max _{\alpha_{i}, \beta_{i}} \overline{\mathcal{C}}=c \sum_{i=1}^{N} \alpha_{i} \cdot g^{(i)}(\vec{\beta}) \\
& \text { subject to }\left\{\begin{array}{l}
\sum_{i=1}^{N} \alpha_{i}=1, \alpha_{i} \geq 0 \\
\prod_{i=1}^{N} \beta_{i}=\delta^{2}, \beta_{i} \leq 1
\end{array}\right.
\end{aligned}
$$

where $g^{(i)}(\vec{\beta})=\left(1-\beta_{i}\right)\left(\prod_{j=1}^{i} \beta_{j}\right)^{(\rho-2) / 2}$ is a function of $\vec{\beta}=\left(\beta_{1}, \cdots, \beta_{N}\right)$ and $c=\frac{P_{0}}{N_{0}}\left(\frac{d_{0}}{\delta}\right)^{\rho} \frac{\delta^{2}}{1-\delta^{2}}$ is a constant. Let $\vec{\beta}^{*(i)}=\left(\beta_{1}^{*(i)}, \cdots, \beta_{N}^{*(i)}\right)$ be the optimal solution to the following maximization problem

$$
\begin{aligned}
& \max _{\beta_{i}} \overline{\mathcal{C}}^{(i)}=g^{(i)}(\vec{\beta}) \\
& \text { subject to } \prod_{i=1}^{N} \beta_{i}=\delta^{2}, \beta_{i} \leq 1
\end{aligned}
$$

and $g_{i}^{*}=g^{(i)}\left(\vec{\beta}^{*(i)}\right)$ be the optimal value of this problem.

In Theorem 3, we provide the best UGBA scheme of throughput maximization. First an inequality needed in the proof of this theorem is presented.

Lemma 1: $g_{i_{1}}^{*} \geq g_{i_{2}}^{*}$ holds for any $i_{1} \leq i_{2}$.

Proof: For any $i_{1} \leq i_{2}$, we have $\prod_{j=1}^{i_{1}} \beta_{j} \geq \prod_{j=1}^{i_{2}} \beta_{j}$ since $\beta_{j} \leq 1$ holds for any $j$, and $\left(\prod_{j=1}^{i_{1}} \beta_{j}\right)^{(\rho-2) / 2} \geq$ $\left(\prod_{j=1}^{i_{2}} \beta_{j}\right)^{(\rho-2) / 2}$ since typically $\rho \geq 2$. This leads to the inequality $\max _{\beta_{j}}\left(1-\beta_{i_{1}}\right)\left(\prod_{j=1}^{i_{1}} \beta_{j}\right)^{(\rho-2) / 2} \geq \max _{\beta_{j}}(1-$ $\left.\beta_{i_{2}}\right)\left(\prod_{j=1}^{i_{2}} \beta_{j}\right)^{(\rho-2) / 2}$, because $\beta_{i_{1}}$ and $\beta_{i_{2}}$ have the same numerical range. So we have $g_{i_{1}}^{*} \geq g_{i_{2}}^{*}$ for any $i_{1} \leq i_{2}$.

Theorem 3: To maximize the user's average throughput, the entire system bandwidth is allocated to the unique group with its radial location ranging from $\delta$ to $r^{*}$ presented in (10).
TABLE I

UTILITY COMPARISON FOR $\kappa=0.1,0.15,0.2,0.25,0.3$

\begin{tabular}{|c|c|c|c|c|c|}
\hline$\kappa$ & 0.1 & 0.15 & 0.2 & 0.25 & 0.3 \\
\hline$\overline{\mathcal{U}}_{3}^{*(1)}$ & 0.664 & 0.761 & 0.815 & 0.849 & 0.872 \\
\hline$\overline{\mathcal{U}}_{3}^{*(2)}$ & 650631.55 & 1546.07 & 79.14 & 14.31 & 5.09 \\
\hline$\overline{\mathcal{U}}_{3}^{S(2)}$ & 650631.28 & 1546.07 & 79.14 & 14.31 & 5.09 \\
\hline$\overline{\mathcal{U}}_{3}^{*(3)}$ & 651625.09 & 1570.89 & 83.15 & 15.58 & 5.61 \\
\hline$\overline{\mathcal{U}}_{3}^{S(3)}$ & 651625.11 & 1570.82 & 83.15 & 15.58 & 5.61 \\
\hline$\overline{\mathcal{U}}_{3}^{S(4)}$ & 651626.43 & 1571.11 & 83.20 & 15.58 & 5.61 \\
\hline
\end{tabular}

Proof: If the system bandwidth is allocated among several MGs with increasing numbering $i_{j}(j=1, \cdots, L)$, we have $\sum_{j=1}^{L} \alpha_{i_{j}}=1, \alpha_{i_{j}}>0$. Given any $\vec{\beta}$, the user's average throughput $\overline{\mathcal{C}}$ satisfies $\overline{\mathcal{C}}=c \sum_{j=1}^{L} \alpha_{i_{j}}$. $g^{\left(i_{j}\right)}(\vec{\beta}) \leq c \sum_{j=1}^{L} \alpha_{i_{j}} g_{i_{j}}^{*} \leq c g_{1}^{*}=\overline{\mathcal{C}}_{m}$ where the inequality of Lemma 1 is applied. In order to get the maximal average throughput $\overline{\mathcal{C}}_{m}=c g_{1}^{*}$, all the bandwidth should be allocated to MG 1 . It means $\alpha_{1}=1, \alpha_{i}=0(i \geq 2)$. Meanwhile, the optimal solution of (9) for $i=1$ is

$$
\beta_{1}^{*}=\arg \max _{\beta_{1}}\left(1-\beta_{1}\right) \beta_{1}^{(\rho-2) / 2}=\max \left\{\delta^{2}, \frac{\rho-2}{\rho}\right\} .
$$

The radial location of MG 1 ranges between $\delta$ and $r^{*}$ that equals to

$$
r^{*}=\sqrt{\delta^{2} / \beta_{1}^{*}}=\left\{\begin{array}{ll}
1 & \rho=2 \\
\min \left\{1, \delta \sqrt{\frac{\rho}{\rho-2}}\right\} & \rho>2
\end{array} .\right.
$$

From (10), we get $r^{*}=1$ for $\rho \leq \frac{2}{1-\delta^{2}}$. In this case, all the users requiring the same program in a cell are included in the unique MG and occupy the entire bandwidth allocated to this group so that both of throughput and fairness are guaranteed to all the users. When $\rho>\frac{2}{1-\delta^{2}}$ and then $r^{*}<1$, it's unfair to the users distributed in the area radially ranging from $r^{*}$ to 1 because the correct reception of the data packets can not be assured. Conversely,

$$
\delta \geq \delta^{\prime}=\sqrt{1-2 / \rho}
$$

is requested to obtain $r^{*}=1$. Essentially, $\delta^{\prime}$ implies the maximal radius of the cell wherein both of throughput and fairness are satisfied for each user.

\section{NumERicAl Results}

In this section, numerical simulations are presented to demonstrate the performance of the SGP algorithm and the best UGBA scheme for throughput maximization. We set the reference SNR $P_{0} / N_{0}$ to be $50 d B$ at $d_{0}=0.5 \delta$.

Fig. 2(a) and 2(b) plot the curves of $\overline{\mathcal{U}}_{2}$ versus the fading exponent $\rho$, and $\delta$, respectively, for $N=1,2,3,4$ with $\kappa=2$ and $\delta=0.2$. We observe that in fading environments with $\rho>$ 3.5 , the utility $\overline{\mathcal{U}}_{2}$ significantly decreases with the number of MGs $N$. Therefore, it's better to divide users into more MGs in server fading environments with larger $\rho$. Also, we can see that the larger cell with smaller $\delta$ requires the users to be divided 


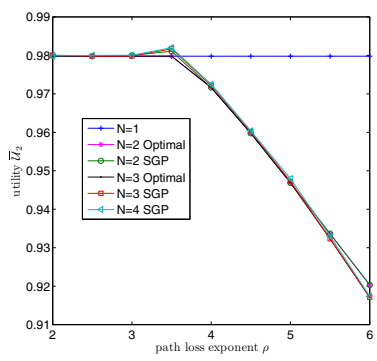

(a) $\kappa=2, \delta=0.2$

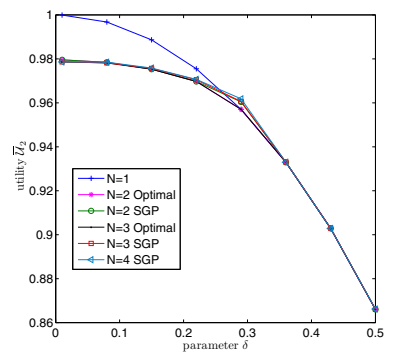

(b) $\kappa=2, \rho=4$
Fig. 2. Comparison of the utility $\overline{\mathcal{U}}_{2}$ for different $N$.

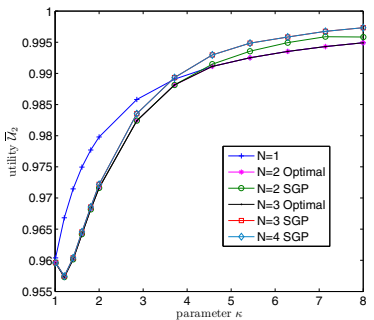

(a) the utility $\overline{\mathcal{U}}_{2}$

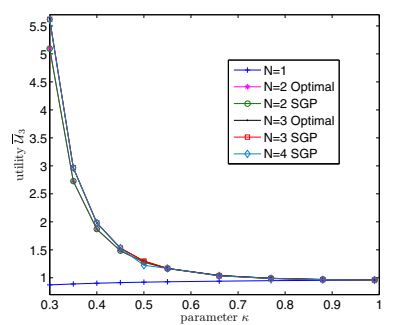

(b) the utility $\overline{\mathcal{U}}_{3}$
Fig. 3. Comparison of $\overline{\mathcal{U}}_{2}\left(\overline{\mathcal{U}}_{3}\right)$ with $\kappa>1(0<\kappa<1)$ for different $N$.

into more MGs. In Fig. 3, two sub-figures illustrate how the utility $\overline{\mathcal{U}}_{2}$ changes with the parameter $\kappa>1$, and $\overline{\mathcal{U}}_{3}$ changes with $0<\kappa<1$ for $\rho=4$ and $\delta=0.2$, respectively. In addition, the complementary numerical results of $\overline{\mathcal{U}}_{3}$ are listed in Table I, wherein $\overline{\mathcal{U}}_{3}^{*(i)}$ and $\overline{\mathcal{U}}_{3}^{S(i)}$ denote the optimal value and the suboptimal value obtained by the SGP algorithm with $N=i$. We see that the one-MG grouping scheme is almost the best for $\kappa>4$ and $\kappa \rightarrow 1$, however, dividing the users into multiple MGs is desirable for small $\kappa(0<\kappa<1)$. From these figures, we can see that the solution achieved by the SGP algorithm is close to the optimal one. Moreover, dividing users into $3 \mathrm{MGs}$ yields a good performance in most cases.

In Fig.4, we plot the optimal outer radius $r^{*}$ of (10) versus the fading exponent $\rho$ for different $\delta$ with y-axis labelling on the left-side, and the parameter $\delta^{\prime}$ of (11) for $r^{*}=1$ versus the fading exponent $\rho$ with y-axis labelling on the right-side. It shows that $r^{*}$ significantly decreases with $\rho$ for certain $\delta$ and increases with $\delta$ for certain $\rho$. When throughput is to be maximized, we can see that the severer fading results in more unfair-users (users with $r>r^{*}$ ). However, the smaller cell with larger $\delta$ leads to relatively fewer unfair-users. Moreover, the red dotted curve in Fig.4 represents the parameter $\delta^{\prime}$ of (11) for $r^{*}=1$ that dramatically increases with $\rho$. It shows that the smaller cell is desirable in more fading environments so as to achieve both throughput and fairness for each user.

\section{CONCLUSION}

In this paper, we investigate the UGBA strategy in wireless multicast systems when the number of users uniformly distributed in the cell and the fixed system bandwidth become

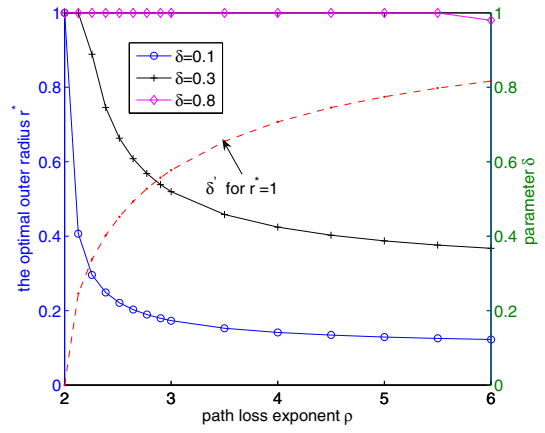

Fig. 4. The optimal radius $r^{*}$ in (10) and the $\delta^{\prime}$ in (11).

sufficiently large. The UGBA problem is formulated into a utility maximization problem that is solved by using a SGP algorithm. The numerical results show that this algorithm works well in finding an appropriate UGBA scheme under different large-scale fadings, throughput-fairness tradeoffs and cell sizes. Through theoretical analysis, we also prove that the best UGBA scheme for throughput maximization is to allocate the entire bandwidth to a group of users, distributed in a ringshaped region characterised by an optimal outer radius.

\section{REFERENCES}

[1] S. Y. Hui and K. H. Yeung, "Challenges in the migration to $4 \mathrm{~g}$ mobile systems," IEEE Commun. Mag., vol. 41, pp. 54-56, December 2003.

[2] Upkar Varshney, "Multicast over wireless networks," Communications of the ACM, vol. 45, pp. 31-37, Dec. 2002.

[3] J. She, F. Hou, P. H. Ho, and L. L. Xie, "IPTV over WiMAX: key success factors, challenges, and solutions," IEEE Commun. Mag., vol. 45, pp. 87-93, Aug. 2007.

[4] L. J. Cimini and N. R. Sollenberger, "OFDM with diversity and coding for advanced cellular internet services," in Proc. IEEE Globecom, Nov. 1997, pp. 305-309.

[5] T. M. Cover, "Broadcast channels," IEEE Trans. Inform. Theory, vol. IT-18, no. 1, pp. 2-14, Jan. 1972.

[6] L. Li and A. Goldsmith, "Capacity and optimal resource allocation for fading broadcast channels. i. ergodic capacity," IEEE Trans. Inform. Theory, vol. 47, no. 3, pp. 1083-1102, 2001.

[7] N. Jindal and A. Goldsmith, "Capacity and optimal power allocation for fading broadcast channels with minimum rates," IEEE Trans. Inform. Theory, vol. 49, pp. 2895 - 2909, Nov. 2003.

[8] M. Sharif, A. F. Dana, and B. Hassibi, "Differentiated rate scheduling for gaussian broadcast channels," in Proc. IEEE International Symp. Inform. Theory, Sep. 2005, pp. 2179-2183.

[9] H. Won, Han Cai, D. Y. Eun, K. Guo, A. Netravali, I. Rhee, and K. Sabnani, "Multicast scheduling in cellular data networks," in Proc. IEEE Infocom 2007, Anchorage, Alaska, USA, May 2007.

[10] Changho Suh and Jeonghoon Mo, "Resource allocation for multicast services in multicarrier wireless communications," IEEE Trans. Wireless Commun., vol. 7, no. 1, pp. 27-31, Jan. 2008.

[11] J. Liu, W. Chen, Z. G. Cao, Y. J. (Angela) Zhang, and S. C. Liew, "Asymptotic throughput in wireless multicast ofdm systems," in Proc. IEEE Globecom, New Orleans, LA, USA, Nov. 2008.

[12] F. Kelly, A. Maulloo, and D. Tan, "Rate control for communication networks: shadow price proportional fairness and stability," J. Oper. Res. Soc., vol. 49, pp. 237-252, 1998.

[13] Jeonghoon Mo and Jean Walrand, "Fair end-to-end window-based congestion control," IEEE/ACM Trans. Networking, vol. 8, no. 5, pp. 556-567, Oct. 2000.

[14] S. P. Boyd, S. J. Kim, L. Vandenberghe, and A. Hassibi, "A tutorial on geometric programming," July 2005, [Online]. Available: http://www.stanford.edu//boyd/papers/gp_tutorial.html. 